\title{
Instability inside a rotating gas cylinder subject to axial periodic strain
}

\author{
Y. Duguet, ${ }^{\text {a) }}$ J. F. Scott, and L. Le Penven \\ Laboratoire de Mécanique des Fluides et d'Acoustique, UMR CNRS 5509_Ecole Centrale de Lyon, \\ 69130 Ecully, France
}

(Received 31 March 2005; accepted 2 September 2005; published online 28 November 2005)

\begin{abstract}
We study numerically the instability of a confined rotating gas flow subject to periodic strain along the axis of rotation, under the low Mach number approximation. An axisymmetric time-stepping spectral Galerkin-type code is used to investigate the viscous basic flow and its stability. Parametric resonance can lead to instability of this flow via the growth of inertial modes coupled by the oscillating strain. The marginal stability curve compares well with earlier experimental and (asymptotic) analytical results in the case of the axisymmetric inertial mode $(1,1,0)$. The resulting flow is dominated by a time-oscillating toroidal vortex and differs very little from the theoretical mode. Two different nonlinear regimes are found, one with saturation to a constant modal amplitude, the other with weak periodic modulation. We also show evidence of the presence of an azimuthal circulation, apparently responsible for the observed modulation. (C) 2005 American Institute of Physics. [DOI: 10.1063/1.2130746]
\end{abstract}

\section{INTRODUCTION}

Rotating flows are known to support inertial oscillations whose frequencies are less than twice the basic rotation rate. ${ }^{1}$ These oscillations play a key role in the understanding of the dynamics of rotating flows, especially for the long-standing issue of flow breakdown commonly observed in experiments. However, the study of such inertial modes in confined geometries is made difficult by the fact that these inertial modes decay monotonically under the action of molecular viscosity, hence they are not observable on a long time scale. For the past few decades, several experiments have shown evidence of the existence of such modes forced either directly or by means of an indirect resonance mechanism. The former case refers to studies in a rotating closed cylinder subject to precession effects. ${ }^{2,3}$ The latter concerns essentially the phenomenon known as "elliptic instability," where the basic flow is rotating in a cylindrical geometry slightly deformed in an elliptical way by imposing small eccentricity to the boundaries in the plane orthogonal to the axis of rotation. $^{4-6}$ Seen from the rotating frame, the ellipticity of the boundaries is equivalent to a lateral deformation rate which is applied periodically in time. In this configuration, the instability manifests itself through the growth of a pair of nonaxisymmetric inertial modes resonantly coupled by the elliptical distortion. ${ }^{6}$ Nonlinear evolution leads to a sudden breakdown of the flow and a disorderly, apparently turbulent regime. Subsequently, the flow relaminarizes, the initial instability kicks back in, and a cycle of disorderly and orderly phases is observed. $^{7}$

Here we propose a mechanism for the growth of axisymmetric modes by applying to a uniformly rotating flow a deformation rate whose principal direction is parallel to the rotation axis. This configuration was first studied by Man-

\footnotetext{
a) Author to whom correspondence should be addressed. Telephone: +33472186189. Fax: +33478647145. Electronic mail: yohann.duguet@ec-lyon.fr
}

sour and Lundgren. ${ }^{8}$ They performed a linear stability analysis of such a flow under the assumption of low Mach number, and in the absence of walls and viscosity. Their analysis revealed that the flow is generally unstable when the forcing frequency $\omega$ lies in the range $[0: 4 \Omega]$, where $\Omega$ stands for the rotation rate. We examine the case of viscous flow inside a closed circular cylinder rotating about its axis, periodically compressed by means of a piston (see Fig. 1). The piston is supposed to rotate at the same angular frequency as the cylinder itself. Piston motion thus induces time variations of the height $h(t)$ of the cylinder as $h(t)=h_{0}\left(1+\epsilon \cos \omega_{0} t\right)$, where $h_{0}$ is the mean height of the cylinder, $\omega_{0}$ is the angular frequency of the forcing, and $\epsilon$ is a parameter measuring the amplitude of the forcing [note that space and time are nondimensionalized, respectively, by the radius $a$ of the cylinder and by the rotation time $\left.(2 \Omega)^{-1}\right]$. In the absence of viscosity, the basic flow corresponding to such a configuration is an axial oscillation of the fluid in the rotating frame with frequency $\omega_{0}$. Adding small viscosity to the problem, no analytical solution can be found. Nevertheless, we expect the corresponding basic flow to be also oscillatory with frequency $\omega_{0}$, with velocity gradients confined to thin $O\left(\mathrm{Re}^{-1 / 2}\right)$ boundary layers. A complete stability analysis of this basic flow was performed by $\mathrm{Racz}^{9}$ under the assumption of asymptotically small $\epsilon$ and large $\operatorname{Re}=O\left(\epsilon^{-2}\right)$, where $\mathrm{Re}$ $=2 \Omega a^{2} / \nu$ is a Reynolds number based on the rotation rate. Linear stability analysis leads to a parametric resonance condition: two inertial modes $\mu$ and $\nu$ can be coupled by the oscillation of the piston if their angular frequencies, respectively $\omega^{(\mu)}$ and $\omega^{(\nu)}$, obey the following relation:

$$
\left|\omega^{(\mu)}-\omega^{(\mu)}\right|=\omega_{0}+O(\epsilon) .
$$

This relation provides an inertial mode selection criterion which allows, by carefully selecting the forcing frequency, to destabilize a chosen pair of inertial modes. It is consistent with Mansour and Lundgren's prediction, except that the potentially unstable modal frequencies only form a 


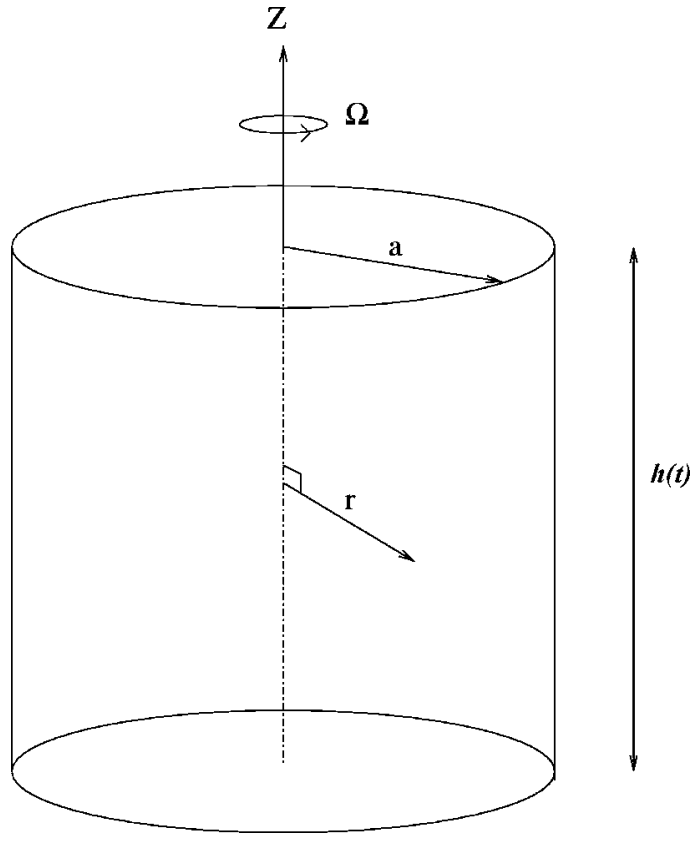

FIG. 1. Cylindrical configuration with notation.

discrete but dense set in $[0: 4 \Omega]$ rather than a continuum of frequencies. Viscosity acts both as a high wave-number filter (hence reducing the density of modes) and as a stabilizing effect, so that for each mode pair there is a critical piston amplitude, decreasing with increasing rotation rate, below which the basic flow is always stable. In the case of axisymmetric modes, Racz's analysis shows that one given inertial mode $\mu$ of frequency $\omega^{(\mu)}$ can only be linearly coupled with its conjugate mode $\mu^{*}$ of frequency $\omega^{\left(\mu^{*}\right)}=-\omega^{(\mu)}$, so that a potentially unstable pair of axisymmetric modes represents only one real physical entity, leading to a subharmonic response at exactly half the forcing frequency. For an $O(1)$ mean aspect ratio of the cylinder, the most unstable pair of inertial modes is generally an axisymmetric one, so it appears worth restricting our attention to an axisymmetric configuration first. Weakly nonlinear theory using an expansion of the modal amplitudes in powers of $O\left(\epsilon^{1 / 2}\right)$ showed that the amplitude of the most unstable mode pair saturates at order of magnitude $O\left(\epsilon^{1 / 2}\right)$, hence dominating the piston oscillations, which are of order $O(\epsilon)$. Nonlinear mode-mode coupling generates $\omega=0$ and $\omega=2 \omega^{(\mu)}$ frequencies of order $O(\epsilon)$. Among these are geostrophic modes $(\omega=0)$, which modify the mean flow. Several kinds of dynamics are predicted according to the values of the parameters, ranging from stability of the basic flow through instability with saturation of the modal amplitude, to amplitude modulation and even divergence. Complementary to this analytical work, an experimental study was carried out by Graftieaux ${ }^{10,11}$ using a rotating glass cylinder along with laser Doppler anemometry (LDA) and particle image velocimetry (PIV) measurements. Experimental data reproduce well the main features of the instability mechanism predicted analytically.

Guided by these former works, the purpose of this study is to perform a numerical simulation of the configuration studied by Racz in order to describe the principal features of the instability. We focus attention on the most unstable axisymmetric mode corresponding to the aspect ratio of the cylinder used in Graftieaux's experiment. Our objective is a better comprehension of the spatial structure of the basic flow, a determination of the instability regions in parameter space, a review of the possible long-time dynamics of the flow, and a discussion of the relevance of the basis of inviscid modes for the theoretical description of the dynamics.

The plan of this paper is as follows. Section II introduces the mathematical formulation of the problem under a low Mach approximation, including the change of coordinates, which enables use of a truncated Galerkin expansion scheme for the numerical simulation. Section II C describes the numerical technique used with details about the special treatment of the flow singularities in the corners. Section III presents the results that are then discussed in Sec. IV.

\section{FORMULATION}

\section{A. Equations of the problem}

We consider the motion of a viscous fluid inside a closed circular cylinder of radius $a$ and height $h(t)$, one of whose extremities oscillates axially by means of a piston, the flow domain being $r<a, 0<z<h(t)$. The whole setup is rotating at constant angular velocity $\Omega$ about the $z$ axis, which coincides with the cylinder axis. We assume that the Mach number $M$ based on both the rotation rate and piston frequency is small. In addition, we assume for simplicity sake that the walls are adiabatic and that the initial conditions are homogeneous. These assumptions ${ }^{9}$ enable us to decouple the thermodynamic aspects of the problem and to consider at leading order in $O\left(M^{2}\right)$ a viscous flow with spatially homogeneous (but time-dependent) variables such as density, temperature, and viscosity. From here on, we nondimensionalize all quantities using the cylinder radius $a$ as length scale and $(2 \Omega)^{-1}$ as time scale. The equations of motion in the rotating frame, expressed in the velocity-pressure formulation $(\boldsymbol{v}, p)$, read

$$
\begin{aligned}
& \boldsymbol{\nabla} \cdot \boldsymbol{v}=\frac{\dot{h}}{h}, \\
& \frac{\partial \boldsymbol{v}}{\partial t}+(\boldsymbol{v} \cdot \boldsymbol{\nabla}) \boldsymbol{v}=-\boldsymbol{\nabla} p+\frac{1}{R(t)} \nabla^{2} \boldsymbol{v}-\boldsymbol{e}_{z} \times \boldsymbol{v},
\end{aligned}
$$

where $R(t)=2 \Omega a^{2} / \nu(t)$ is a time-dependent Reynolds number, which reflects the time-varying kinematic viscosity $\nu(t)$, and $\boldsymbol{e}_{z}$ is the unit vector in the $z$ direction. Along with these equations, there are viscous boundary conditions expressing no-slip at the fixed and moving boundaries,

$$
\begin{aligned}
& \boldsymbol{v}=\mathbf{0} \quad(r=1), \\
& \boldsymbol{v}=\mathbf{0} \quad(z=0), \\
& \boldsymbol{v}=\frac{d h}{d t} \boldsymbol{e}_{z} \quad(z=h(t)) .
\end{aligned}
$$

The nondimensionalized height of the cylinder is assumed to vary according to the harmonic law $h(t)=h_{0}(1$ $\left.+\epsilon \cos \omega_{0} t\right)$. In order to deal with a constant parameter, we 
use the Reynolds number based on the value of the viscosity for the nonmoving piston case $\operatorname{Re}=2 \Omega a^{2} /\left.\nu\right|_{\epsilon=0}$, which corresponds roughly to the mean value of $R(t)$. The number of independent nondimensional parameters is thus brought to four: the mean Reynolds number $\mathrm{Re}$, the mean geometrical aspect ratio of the cylinder $h_{0}$, the angular forcing frequency $\omega_{0}$, and the forcing amplitude $\epsilon$.

\section{B. Inertial waves}

Let us consider, in the case $\epsilon=0$ of nonmoving boundaries, the inviscid version of the system (2) and (3) linearized around the equilibrium state $\boldsymbol{v}=0, p=p_{0}$. This problem admits separable solutions ${ }^{1}$ of the form $\left(\boldsymbol{v}, p-p_{0}\right)$ $=\left(\boldsymbol{u}^{(\mu)}, \lambda^{(\mu)}\right) e^{-i \omega^{(\mu)} t}$, where for a given solution,

$$
\begin{aligned}
& u_{r}(\boldsymbol{x})=i N\left(\omega k J_{n}^{\prime}(k r)-\frac{n}{r} J_{n}(k r)\right) \cos \left(\frac{m \pi z}{h_{0}}\right) e^{i n \theta}, \\
& u_{\theta}(\boldsymbol{x})=N\left(k J_{n}^{\prime}(k r)-\frac{n \omega}{r} J_{n}(k r)\right) \cos \left(\frac{m \pi z}{h_{0}}\right) e^{i n \theta}, \\
& u_{z}(\boldsymbol{x})=i N \frac{\omega k^{2} h_{0}}{m \pi} J_{n}(k r) \sin \left(\frac{m \pi z}{h_{0}}\right) e^{i n \theta}, \\
& \lambda(\boldsymbol{x})=N\left(1-\omega^{2}\right) J_{n}(k r) \cos \left(\frac{m \pi z}{h_{0}}\right) e^{i n \theta} .
\end{aligned}
$$

Here, the angular frequency $\omega^{(\mu)}$ and radial wave number $k^{(\mu)}$ of each mode can be obtained from

$$
\begin{aligned}
& \omega= \pm\left[1+\left(\frac{k h_{0}}{m \pi}\right)^{2}\right]^{-1 / 2}, \\
& \omega k J_{n}^{\prime}(k)=n J_{n}(k) .
\end{aligned}
$$

For given values of the integers $m$ and $n$, there is a discrete infinity of $\omega^{(\mu)}, k^{(\mu)}>0$, which may be indexed by an integer $\alpha=1,2, \ldots$ so that the $k^{(\mu)}$ are in increasing order. These eigenmodes of the unforced inviscid system, called inertial modes or sometimes Kelvin modes, are the inviscid counterpart of the viscous structures which appear spontaneously through an instability mechanism. They form an orthogonal basis indexed by the integer multilabel $\mu=(\alpha, m, n)$, where $\alpha, m$, and $n$ are related to the number of radial, axial, and azimuthal nodes. The coefficient $N^{(\mu)}$ is used to normalize $\boldsymbol{u}^{(\mu)}$ with respect to the $L^{2}$ norm. In the axisymmetric case, corresponding to the value $n=0$, inviscid inertial modes consist of an array of oscillating vortices of fixed toroidal form. The degenerate family $n=0, m=0$ corresponds to pure azimuthal motion, unsteady and independent of the axial variable $z$. Such inertial modes are called geostrophic. Note that the corresponding eigenvalue problem in the presence of viscosity has no analytical solution, and that the related viscous eigenmodes do not form an orthogonal basis, thus they are not the easiest quantities to be handled analytically for the purpose of this study. For asymptotically large values of Re, differences between the viscous modes and the related inviscid ones are effectively localized near the boundaries in Ekman layers of thickness $O\left(\mathrm{Re}^{-1 / 2}\right)$. Outside these layers, the modifications due to viscosity represent a weak secondary flow of relative magnitude $O\left(\mathrm{Re}^{-1 / 2}\right) .{ }^{12}$ Angular frequencies are complex, the imaginary part representing the $O\left(\mathrm{Re}^{-1 / 2}\right)$ modal viscous decay. Although there is no exact analytical value for these inertial decay rates, asymptotic models exist in the literature, ${ }^{9,13}$ which all yield the same value for the axisymmetric modes, and more precise values can be obtained numerically by solving the related viscous eigenvalue problem. ${ }^{13,14}$ At sufficiently large Reynolds numbers, of order $10^{2}$ for the lowest-order modes, the classification $(\alpha, m, n)$ still holds and Greenspan's asymptotic estimates are valid. ${ }^{14}$

\section{Numerical method}

The time-dependent flow domain $\left\{\boldsymbol{x}=(x, y, z), x^{2}+y^{2}\right.$ $\leqslant 1,0 \leqslant z \leqslant h(t)\}$ is first turned into the fixed one $\{\boldsymbol{X}$ $\left.=(X, Y, Z), \quad X^{2}+Y^{2} \leqslant 1, \quad 0 \leqslant Z \leqslant h_{0}\right\}$ by the coordinate transformation

$$
X=x, \quad Y=y, \quad Z=\frac{h_{0}}{h(t)} z,
$$

and the velocity field $\boldsymbol{v}$ is made divergence-free with respect to the variables $\boldsymbol{X}$ by removing the piston-induced vertical velocity

$$
V_{X}=v_{x}, \quad V_{Y}=v_{y}, \quad V_{Z}=\frac{h_{0}}{h(t)}\left(v_{z}-\frac{z}{h} \frac{d h}{d t}\right) .
$$

Finally, bringing together all terms deriving from a gradient leads to the modified pressure variable

$$
\Pi=p+\frac{1}{2 h} \frac{d^{2} h}{d t^{2}} z^{2} .
$$

The system of partial differential equations describing the evolution of $\boldsymbol{V}(\boldsymbol{X}, t)$ now reads

$$
\begin{aligned}
\boldsymbol{\nabla} \cdot \boldsymbol{V}=0, & \\
\frac{\partial \boldsymbol{V}}{\partial t}+(\boldsymbol{V} \cdot \boldsymbol{\nabla}) \boldsymbol{V}= & -\boldsymbol{\nabla} \Pi-\boldsymbol{e}_{Z} \times \boldsymbol{V}+\frac{1}{R} \mathcal{D} \boldsymbol{V} \\
& +\boldsymbol{e}_{z}\left(\frac{\partial}{\partial t}+\boldsymbol{V} \cdot \boldsymbol{\nabla}-\frac{1}{R} \mathcal{D}\right)\left[1-\left(\frac{h}{h_{0}}\right)^{2}\right] V_{Z},
\end{aligned}
$$

where all the differential operators refer to the new variable $\boldsymbol{X}$, and the Laplacian operator $\mathcal{D}$ is now expressed by $\mathcal{D}$ $=\partial_{X}^{2}+\partial_{Y}^{2}+\left(h_{0} / h\right)^{2} \partial_{Z}^{2}$. The change of variables leads to a modification of the boundary conditions

$$
\begin{aligned}
& \boldsymbol{V}=-\frac{Z}{h} \frac{d h}{d t} \boldsymbol{e}_{z} \quad(r=1), \\
& \boldsymbol{V}=0 \quad\left(Z=0, h_{0}\right) .
\end{aligned}
$$

The above system of equations was solved using Galerkintype spectral expansion, whose fast convergence properties are well adapted to stability problems such as the one treated here. The main difficulty with spectral methods is that the exponential rate of convergence is only obtainable for infi- 
nitely smooth fields, whereas in practice the order of convergence is proportional to the number of finite derivatives of the solution. ${ }^{15}$ The present problem is singular in the sense that the boundary conditions (18) and (19) impose discontinuity of the velocity components in the piston corner $(r=1$, $Z=h_{0}$ ), as well as discontinuity of the first spatial derivatives of the velocity in the other corner $(r=1, Z=0)$. In practice, this singularity is resolved by the presence of a very thin gap between the piston and the side wall, eliminating the infinite peak of pressure, ${ }^{16}$ but the correct representation of the flow inside the gap is a difficult task that is beyond the scope of this paper. Numerical algorithms such as finite volume, finite difference, or Chebyshev-Tau methods treat the singularity problem by locally smoothing the discontinuities in the boundary conditions, ${ }^{17}$ which act as a local model for the dynamical behavior in the vicinity of the gap region. In contrast to these techniques, the method used in this study is aimed at solving the singular problem itself instead of another regularized well-posed problem. The main idea is to split the velocity field $\boldsymbol{V}$ in two parts. The first part, called the singular part, is constructed analytically so that it possesses exactly the same boundary conditions as $\boldsymbol{V}$, and mimics the singularity at the cylinder corner. The regularity of the second part allows an efficient use of a Galerkin spectral scheme. This decomposition reads

$$
\boldsymbol{V}(\boldsymbol{X}, t)=-\frac{\dot{h}(t)}{h(t)} \boldsymbol{V}_{0}(\boldsymbol{X})+\sum_{\mu} A^{\mu}(t) \phi^{\mu}(\boldsymbol{X}) .
$$

The singular field $\boldsymbol{V}_{0}$ is constructed by interpolation of local solutions of the viscous linear Stokes problem $\boldsymbol{\nabla}$ $\times \nabla^{2} \boldsymbol{v}=\mathbf{0}$ in each corner region with adequate singular boundary conditions. ${ }^{18}$ This technique makes sure that the singular part respects the structure of the real solution $\boldsymbol{V}$ in the spatial regions sufficiently near the corners, where the assumption of a Stokes flow holds. Note that since the singular part in Eq. (20) satisfies the boundary conditions (18) and (19), the spectral expansion represents a velocity field which is zero at the walls. The meridian components of the divergence-free basis functions $\left(\phi^{\mu}\right)_{\mu=(n, m)}$ derive from a meridian streamfunction $\psi^{\mu}$ using the formula

$$
\phi_{r}^{\mu}=\frac{1}{r} \frac{\partial \psi^{\mu}}{\partial Z}, \quad \phi_{Z}^{\mu}=-\frac{1}{r} \frac{\partial \psi^{\mu}}{\partial r} .
$$

Both $\phi_{\theta}^{\mu}$ and $\psi^{\mu}$ are products of Chebyshev polynomials $T_{n}(r)=\cos \left[n \cos ^{-1}(r)\right] \quad$ and $T_{m}\left(2 Z / h_{0}-1\right)$ $=\cos \left[m \cos ^{-1}\left(2 Z / h_{0}-1\right)\right]$ ( $n$ and $m$ being positive integers), known for their property to represent economically strong gradients in near-wall regions. They are weighted with polynomial prefactors chosen so that the basis functions exactly satisfy the boundary conditions of zero velocity. Their expression reads

$$
\psi^{(n, m)}= \begin{cases}0 & (n \text { odd }) \\ r^{2}\left(1-r^{2}\right)^{2} Z^{2}\left(Z-h_{0}\right)^{2} T_{n}(r) T_{m}\left(\frac{2 Z}{h_{0}}-1\right) & (n \text { even })\end{cases}
$$

$$
u_{\theta}^{(n, m)}= \begin{cases}\left(1-r^{2}\right) Z\left(Z-h_{0}\right) T_{n}(r) T_{m}\left(\frac{2 Z}{h_{0}}-1\right) & (n \text { odd }) \\ 0 & (n \text { even }) .\end{cases}
$$

Projection of Eqs. (17) onto the divergence-free functional basis defined above transforms the problem into a dynamical system for the spectral amplitudes $A^{(n, m)}(t)$ of the form

$$
\begin{aligned}
\frac{d}{d t} \sum_{\nu} \beta^{\mu \nu}(t) A^{\nu}(t)= & \sum_{\nu}\left(\frac{1}{R(t)} D^{\mu \nu}(t)+C^{\mu \nu}\right) A^{\nu}(t)+N^{\mu}(t) \\
& +f^{\mu}(t)
\end{aligned}
$$

where $C$ and $D$ stand, respectively, for the linear spectral time-dependent operators representing viscous diffusion and rotation effects, $f$ expresses the forcing by the singular field (which is zero in the absence of piston motion), and $N$ is the nonlinear term. The infinite sum is truncated by retaining only the values of $n$ and $m$ up to a given value $M$. The spectral coefficients as well as the nonlinear term are evaluated in physical space using a two-dimensional (2D) Romberg integration scheme. Then the solution vector $\boldsymbol{A}(t)$ is advanced in time using an implicit Adams-Moulton scheme for the forcing term and the linear term, and an explicit Adams-Bashforth scheme for the nonlinear term. Time discretization using a finite time step $\Delta t$ reduces the integration of the dynamical system in time to the linear system

$$
\begin{aligned}
\left(\beta^{n+1}-\frac{5}{12} \Delta t L^{n+1}\right) A^{n+1}= & \left(\beta^{n}+\frac{8}{12} \Delta t L^{n}\right) A^{n}-\frac{\Delta t}{12}\left(L^{n-1} A^{n-1}\right) \\
& +\frac{\Delta t}{12}\left(5 f^{n+1}+8 f^{n}-f^{n-1}\right) \\
& +\frac{\Delta t}{2}\left(3 N^{n}-N^{n-1}\right)
\end{aligned}
$$

Lack of symmetry as well as the nonsparse structure of the operator on the left-hand side lead to the use of the BiCG-Stab algorithm for the inversion of the system at every time step. The nonorthogonality of the functional basis with respect to the $L^{2}$ scalar product makes the matrix $\beta^{\mu \nu}(t)$ $=\int\left[\boldsymbol{\phi}_{r}^{\nu} \boldsymbol{e}_{r}+\boldsymbol{\phi}_{\theta}^{\nu} \boldsymbol{e}_{\theta}+\left(h / h_{0}\right)^{2} \boldsymbol{\phi}_{Z}^{\nu} \boldsymbol{e}_{Z}\right] \cdot \boldsymbol{\phi}^{\mu} d^{3} X$ ill-conditioned. Solving the system (25) at every time step is made possible by using a constant preconditioning by $\beta_{0}^{-1}=\left.\beta(t)\right|_{\epsilon=0}$, in other words by the Gram-Schmidt matrix related to the functional basis. Convergence was verified by doubling the resolution, revealing that $M=32$ and 400 time steps per piston period proved sufficient for $\operatorname{Re}<2 \cdot 10^{4}$.

\section{RESULTS}

A numerical investigation of the four-dimensional parameter space has been carried out for an aspect ratio $h_{0}$ $=1.18$ and a piston displacement $\epsilon=0.131$. This was motivated by the experimental conditions initially chosen by Graftieaux. In this configuration, the most unstable mode (stricto senso a pair of conjugate axisymmetric modes) is $(1,1,0)$, whose inviscid angular frequency $\omega^{1,1,0}$ is close to 


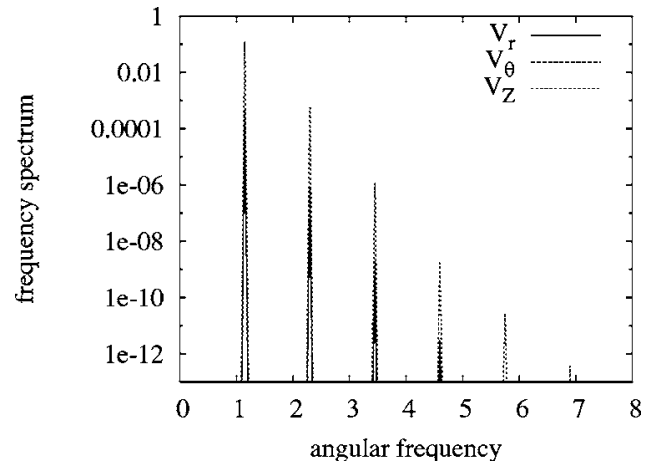

FIG. 2. Frequency spectrum for each velocity component of the basic flow, at $(r, Z)=\left(0.094, h_{0} / 2\right)$ for $\omega_{0}=1.05$ and $\operatorname{Re}=1000$, after the transient phase.

0.57. Starting from an initial flow at rest inside the cylinder, long-time calculations were performed with forcing at various angular frequencies $\omega_{0}$ mostly close to $2 \omega^{1,1,0}$, motivated by Racz's analysis.

\section{A. Basic flow}

For Re less than a critical value a little above 5000 or values of $\omega_{0}$ sufficiently far from $2 \omega^{1,1,0}$, the frequency spectra associated with every velocity component always had the same qualitative form. All the spectra are dominated by a strong peak at dominant angular frequency $\omega_{0}$ and include higher-order harmonics, as shown in Fig. 2. This corresponds to a periodic flow of period $2 \pi / \omega_{0}$ after a transient phase of about 20 piston cycles. Outside the boundary layers, the azimuthal component $v_{\theta}$ is very nearly in phase quadrature with $v_{Z}$ and $v_{r}$, a classic illustration of Coriolis effects in rotating flows. This $\omega_{0}$-periodic flow is the axisymmetric basic flow whose instability is the subject of this paper.

The basic flow is known analytically only under the assumption of zero viscosity, in which case in the rotating frame it consists of an axial oscillation induced by piston motion at frequency $\omega_{0}$ and with velocity $\dot{h} / h z \boldsymbol{e}_{z}$. The axial velocity component is plotted in Fig. 3, which also shows numerical and experimental results of Graftieaux. ${ }^{11}$ Note that the near-wall structure of the flow is not available by experimental means. All velocity profiles coincide until the distance to the wall is smaller than $1 \%$ of the cylinder radius, reflecting the presence of an inviscid core and the sidewall

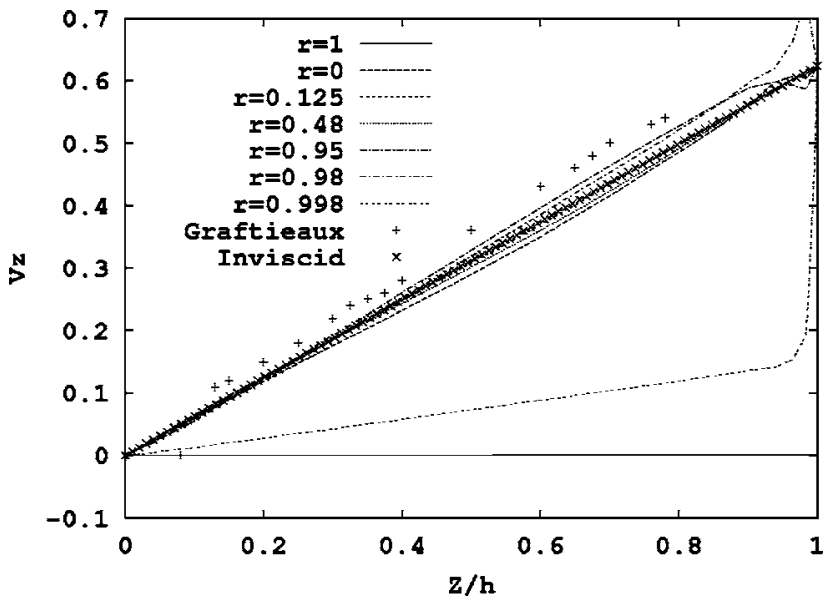

FIG. 3. Axial velocity profile as a function of $z / h$ for several values of $r$. Comparison with the inviscid and the experimental profile by Graftieaux (Ref. 11) at $r=0 . \quad a=70 \mathrm{~mm}, \quad h_{0}=1.18, \quad \epsilon=0.131, \quad \omega_{0}=1.523, \Omega$ $=18.85 \mathrm{rad} \mathrm{s}^{-1}, \mathrm{Re}=9989$.

boundary layer. The velocity discontinuity at the piston corner induces a vorticity line source, giving rise to a localized toroidal corner vortex (see Fig. 4). Whereas the sense of rotation of the vortex changes periodically in time, its position and size do not vary and no ejection phenomena were noticed. The oscillatory boundary layer along the lateral wall $r=1$ can be thought of as an oscillatory Stokes layer since the Coriolis force vanishes for flows mainly parallel to the rotation axis. A vortical secondary flow of magnitude weaker than the corner vortex is found outside the near-wall regions, as well as a weak unsteady Ekman layer on top and bottom of the cylinder. These Ekman layers are characterized by an angular deviation of the velocity components near the boundary. Note that the forcing angular frequency $\omega_{0}$ $\sim 2 \omega^{(1,1,0)} \sim 1.15$ is greater than 1 , which excludes direct resonant excitation of inertial modes, since their frequencies are bounded by unity. The case $\omega_{0}<1$ induces a direct response of the flow in term of inertial waves along conical jets following characteristic surfaces. ${ }^{14}$ The study of such a flow and its consequences on the instability mechanism will be discussed in a future paper.

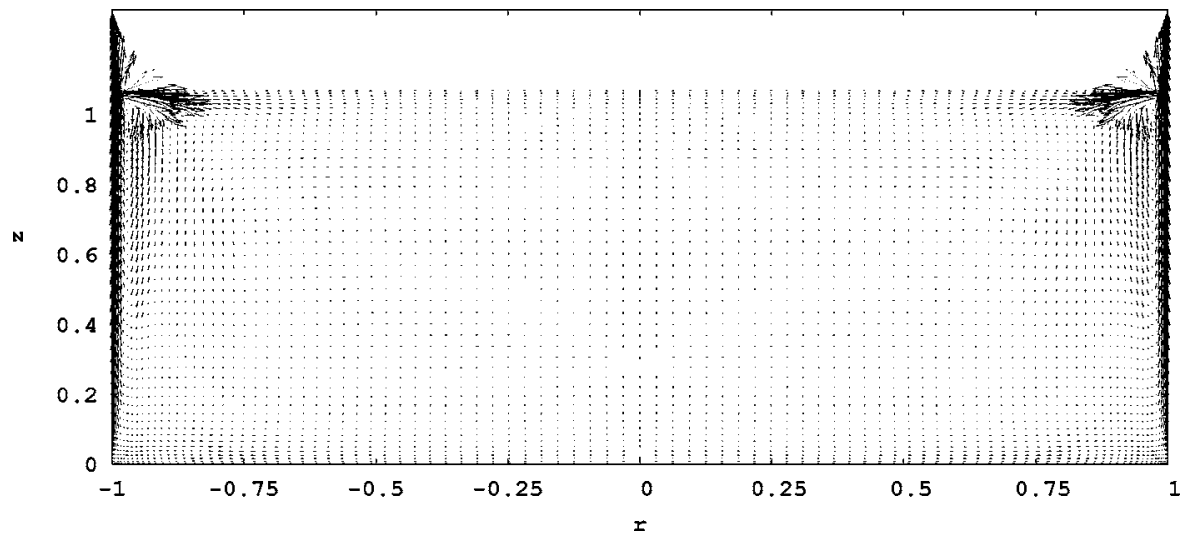

FIG. 4. Plane through the cylinder axis showing the instantaneous difference between the viscous and inviscid basic flow $\left(h_{0}=1.18, \epsilon=0.131, \operatorname{Re}\right.$ $=9989, \omega_{0}=1.523$. 

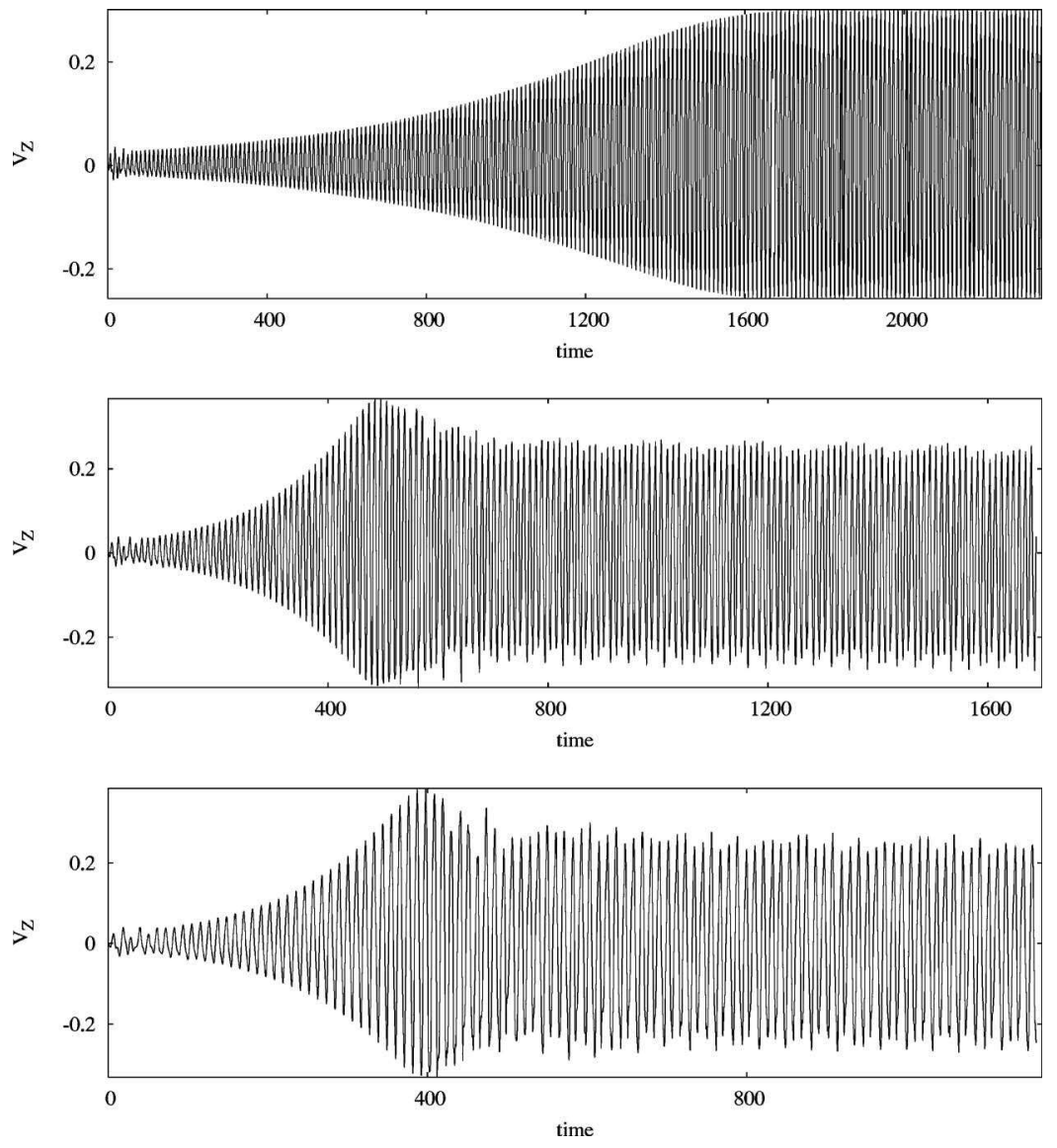

FIG. 5. Axial velocity at $(r, Z)=\left(0.094, h_{0} / 2\right)$ for $\omega_{0}$ $=1.15$ and $\operatorname{Re}=6000$ (top), $\mathrm{Re}=8500$ (middle), and $\mathrm{Re}$ $=10000$ (bottom).

\section{B. Instability of the mode $(1,1,0)$}

For values of Re above the critical threshold and $\omega_{0}$ sufficiently near the resonant value $2 \omega^{(1,1,0)}$, the frequency spectra of all three velocity components clearly show the appearance of a dominant peak at angular frequency $\omega_{0} / 2$, whereas the distribution of the spectral amplitudes of the harmonics of the piston is very similar to the basic flow. Plots of velocity versus time show a slow exponential growth of the envelope of the signal while the flow oscillates rapidly at angular frequency $\omega_{0} / 2$ (see Fig. 5). This slow growth regime can be interpreted as a linear instability regime of the basic flow studied in the previous section. After a long transient phase, whose duration is strongly dependent on the value of the parameters $\operatorname{Re}$ and $\omega_{0}$, the oscillations attain a saturation amplitude. For values of Re sufficiently close to the instability threshold, the envelope saturates at a constant amplitude, as in the case $\operatorname{Re}=6000$ (see top of Fig. 5). A slight increase of $R e$ leads to a weak periodic modulation of the envelope, indicating a possible Hopf bifurcation (see Fig. 5). Simulations were performed up to $\mathrm{Re}=12000$, and had similar characteristics to the case shown here. No case of amplitude divergence has been observed.

\section{Linear stability and the neutral curve}

The Prony algorithm ${ }^{19}$ was applied to the velocity data for the exponential transient phase to calculate the complex exponential growth rates $\sigma$. Among the values of $\sigma$ given by the algorithm, there was a cluster very near to $\operatorname{Im}(\sigma)=\omega_{0} / 2$, of which the one having the largest real part was selected.
This value of $\operatorname{Re}(\sigma)$ was interpreted as the linear growth rate of the instability. The results are illustrated in Table I. Numerical interpolation of these data enables reconstruction of a marginal stability curve in the parameter space $\left(\omega_{0}-\mathrm{Re}\right)$, corresponding to $\operatorname{Re}(\sigma)=0$. The marginal curve takes the form of a narrow instability tongue centered on $2 \omega^{1,1,0}$ and separating stable regions (Re below the curve) from unstable ones ( $\operatorname{Re}$ above the curve). Figure 6 shows the comparison between the numerical marginal curve, the experimental one, and the analytical one, derived from an assumption of asymptotically small $\epsilon$. ${ }^{9}$ The correspondence between the theoretical and the numerical estimates is striking given that $\epsilon$ $=0.131$ seems perhaps at first glance too large a value to ensure the validity of the asymptotic theory. The departure between critical instability thresholds does not exceed 3\% for Re and $0.4 \%$ for $\omega_{0}$. Quantitative departure from the experimental results appears larger, of order $17 \%$. It has not yet been possible to identify the precise reasons for this. However, it may be noted that experimental studies for supercritical bifurcations typically overestimate the stability threshold because the modal amplitude is too small to be measurable. Furthermore, the difference could be influenced by thermal effects (not taken into account in the theoretical model) or by a pressure drop in the core of the flow due to possible leakage at the cylinder corner. Nevertheless, all the results in Fig. 6 tend to confirm the hypothesis of a parametric resonance mechanism involving mode coupling by piston motion, responsible for a subharmonic response of the sys- 
TABLE I. Linear growth rate of the inertial mode $(1,1,0)$ extracted from numerical simulation using the Prony algorithm, as a function of the forcing angular frequency $\omega_{0}$ and the Reynolds number Re. $h_{0}=1.18, \epsilon$ $=0.131$.

\begin{tabular}{lccccc}
\hline \hline \multicolumn{1}{c}{$\omega_{0}$} & 1.13 & 1.14 & 1.15 & 1.16 & 1.17 \\
\hline $\operatorname{Re}=4937.5$ & $-2.00 \times 10^{-3}$ & $-8.14 \times 10^{-4}$ & $-6.98 \times 10^{-4}$ & $-1.65 \times 10^{-3}$ & $-3.66 \times 10^{-3}$ \\
$\operatorname{Re}=5375$ & $-1.51 \times 10^{-3}$ & $-3.52 \times 10^{-4}$ & $-2.56 \times 10^{-4}$ & $-1.23 \times 10^{-3}$ & $-3.26 \times 10^{-3}$ \\
$\operatorname{Re}=5812.5$ & $3.81 \times 10^{-4}$ & $1.52 \times 10^{-3}$ & $1.59 \times 10^{-3}$ & $6.09 \times 10^{-4}$ & $-1.43 \times 10^{-3}$ \\
$\operatorname{Re}=6250$ & $1.48 \times 10^{-3}$ & $2.56 \times 10^{-3}$ & $2.57 \times 10^{-3}$ & $1.51 \times 10^{-3}$ & $-6.17 \times 10^{-4}$ \\
$\operatorname{Re}=6687.5$ & $2.46 \times 10^{-3}$ & $3.58 \times 10^{-3}$ & $3.54 \times 10^{-3}$ & $2.34 \times 10^{-3}$ & $-1.59 \times 10^{-5}$ \\
$\operatorname{Re}=7125$ & $3.32 \times 10^{-3}$ & $4.39 \times 10^{-3}$ & $4.31 \times 10^{-3}$ & $3.07 \times 10^{-3}$ & $6.67 \times 10^{-4}$ \\
$\operatorname{Re}=7562.5$ & $4.08 \times 10^{-3}$ & $5.06 \times 10^{-3}$ & $4.89 \times 10^{-3}$ & $3.56 \times 10^{-3}$ & $1.08 \times 10^{-3}$ \\
$\operatorname{Re}=8000$ & $4.83 \times 10^{-3}$ & $5.75 \times 10^{-3}$ & $5.51 \times 10^{-3}$ & $4.11 \times 10^{-3}$ & $1.56 \times 10^{-3}$ \\
\hline \hline
\end{tabular}

tem at half the forcing frequency. The existence of an instability threshold with respect to Re illustrates the stabilizing effect of viscosity.

\section{Saturation and spatial structure}

We now want to determine how closely the flow resulting from instability can be represented by the inertial mode $(1,1,0)$. Focusing on the case $\operatorname{Re}=6000, \omega_{0}=1.15$, as can be seen from the frequency spectra (see Fig. 7), the flow oscillates at various frequencies, among them the frequency of the growing structure at $\omega_{0} / 2$, the piston frequency $\omega_{0}$, and multiple harmonics resulting from nonlinear interactions between these two dominant frequencies. Note that the amplitude of the $\omega_{0} / 2$-peak is considerably higher than those of the piston harmonics, in accord with the small $\epsilon$ asymptotic analysis. ${ }^{9}$ The saturated flow was filtered in frequency space around the frequency $\omega_{0} / 2$ to get rid of other frequency components and transformed back to the time domain. The main effect of filtering is to remove the axial flow directly induced by piston motion as well as the corresponding oscillatory Stokes layer on the lateral surface of the cylinder. Figure 8 shows the filtered velocity field in the meridian plane, the instantaneous height of the cylinder having been respected in order to show the relative phases of the oscillating vortex flow resulting from instability and the piston motion. In contrast to the basic flow where the meridian velocity is in phase with the piston motion, here the meridian velocity field corresponding to the filtered field is out of phase with the piston. The phase difference $\phi$ can be determined from the complex frequency spectra by subtracting the complex argument of the $\omega_{0}$ component from that of the $\omega_{0} / 2$ component,

$$
\phi=\left|\arg \hat{V}_{Z}\left(\omega=\frac{\omega_{0}}{2}\right)-\arg \hat{V}_{Z}\left(\omega=\omega_{0}\right)\right|,
$$

which yields the result $\cos \phi=0.807$. This value is very close to the theoretical result $\cos \phi=0.779$ coming from the analysis of the amplitude equations in Ref. 9 in the case of a fixed point.

The spatial structure of the resulting harmonic flow may be compared with (i) the inertial mode $(1,1,0)$ obtained from (7)-(9) and (ii) the inertial mode $(1,1,0)$ obtained numerically from the viscous eigenvalue problem for the same value of Re. ${ }^{14}$ Let us denote by $\boldsymbol{u}_{F}$ the filtered flow resulting from simulation and $\boldsymbol{u}_{e}$ the complex eigenmode with which it is compared, either viscous or inviscid. The comparison was done by least-squares minimizing the quantity

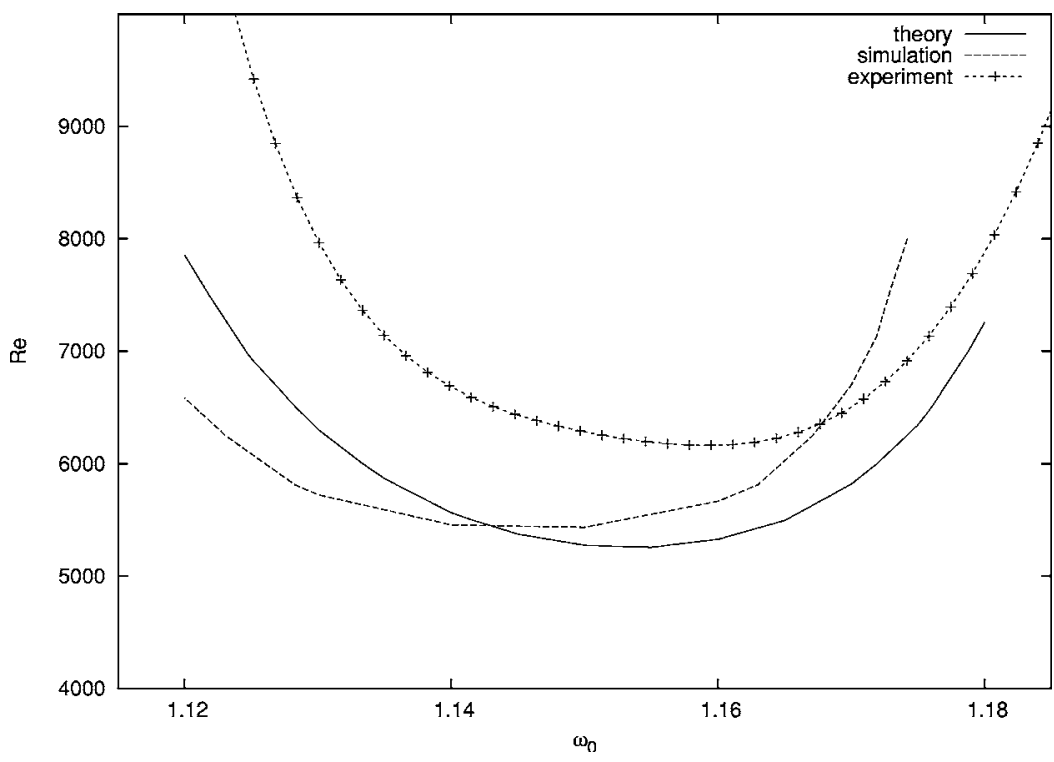

FIG. 6. Marginal stability curve for the inertial mode $(1,1,0)$ for $h_{0}=1.18, \epsilon=0.131$, in the parameter plan $\left(\omega_{0}-\mathrm{Re}\right)$. 


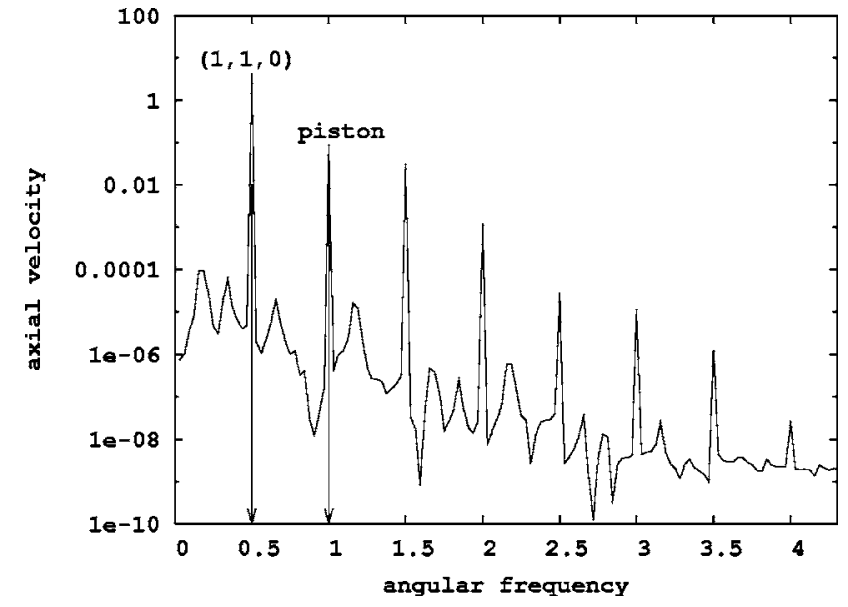

FIG. 7. Frequency spectrum of $V_{Z}\left[r=\left(0.094, Z=h_{0} / 2\right)\right]$, for $\omega_{0}=1.15$ and $\operatorname{Re}=6000$, calculated after removing the transient phase. Angular frequencies are normalized by the piston angular frequency $\omega_{0}$.

$$
\delta=\frac{\int\left|\boldsymbol{u}_{F}-\operatorname{Re}\left(\mathcal{A} \boldsymbol{u}_{e}\right)\right|^{2} d^{3} X}{\int\left|\boldsymbol{u}_{F}\right|^{2} d^{3} X},
$$

with respect to the unknown complex modal amplitude $\mathcal{A}$. Figures 9 and 10 show a comparison between $\boldsymbol{u}_{F}$ and the purely modal flow $\operatorname{Re}\left(\mathcal{A} \boldsymbol{u}_{e}\right)$ and indicate that the inertial mode $(1,1,0)$ is a good approximation of the harmonic flow in the core of the cylinder. The largest departure occurs for $u_{Z}$ in Fig. 10 toward the cylinder axis. A similar result was obtained experimentally. ${ }^{10,11}$ The angular deviation of the velocity field in both the top and bottom Ekman layers is also verified, both their thickness and structure matching those from the viscous mode.

A quantitative measure of the departure from the inviscid mode $(1,1,0)$ was achieved by projection onto the inviscid inertial modes. The related basis set of eigenfunctions is complete in the sense that any solenoidal field $\boldsymbol{u}$ whose normal component vanishes at the boundaries can be expanded in the form

$$
\boldsymbol{u}=\sum_{\mu} B_{\mu} \boldsymbol{u}^{(\mu)},
$$

where the coefficients $B_{\mu}$ can be determined using orthonormality as

$$
B_{\mu}=\int \boldsymbol{u} \cdot \boldsymbol{u}^{(\mu)^{*}} d^{3} \boldsymbol{X} .
$$

The modal energy factor, i.e., the energy fraction of a given mode $\mu$ for a real-valued vector field $\boldsymbol{u}$, can be determined for a nongeostrophic mode by

$$
b^{(\mu)}=\frac{2\left|B_{\mu}\right|^{2}}{\int|\boldsymbol{u}|^{2}} d^{3} \boldsymbol{X}
$$

and for a (real) axisymmetric geostrophic mode $\mu$ by

$$
b^{(\mu)}=\frac{\left|B_{\mu}\right|^{2}}{\int|\boldsymbol{u}|^{2}} d^{3} \boldsymbol{X} .
$$

Note the factor of 2 in formula (30) reflecting the fact that nongeostrophic modes are complex and associated with complex conjugates. Numerical determination of the modal energy factor $b^{(1,1,0)}$ when $\boldsymbol{u}=\boldsymbol{V}(X, t)$ yields a value oscillating between $61 \%$ and $78 \%$ during a piston cycle. When applied to the flow $\boldsymbol{u}_{F}$, the value of $b^{(1,1,0)}$ rises to the constant value $94 \%$. In the case $\mathrm{Re}=8500$, characterized by periodic modulation, an evaluation of the modal energy factor associated with $\boldsymbol{u}_{F}$ yields a value $b^{(1,1,0)}$ oscillating around $94 \pm 0.3 \%$. The optimization of the quantity $\delta$ defined in (27) when $\boldsymbol{u}_{e}$ is the viscous eigenmode $(1,1,0)$ yields approximately $3 \%$. It follows that the flow resulting from instability can be represented by the inviscid mode $(1,1,0)$ with an error of only $6 \%$, and by the viscous mode $(1,1,0)$ with an error of $3 \%$.

The values of the coefficients $b^{(\alpha, m, 0)}$ coming from projection onto other inviscid modes, listed in Table II, show that a large number of weakly energetic modes have roughly similar contributions. This is as predicted analytically, where terms of higher order than the first lead to multimode excitation. In particular, the $O(\epsilon)$ terms do not contain the frequency $\omega_{0} / 2$ and are thus removed by filtering, whereas the $O\left(\epsilon^{3 / 2}\right)$ term has both nonlinear and viscous contributions.

\section{Geostrophic flow}

Whereas all velocity components exhibit a very nearly zero mean value in the case of the basic flow identified in Sec. I, the azimuthal velocity in the unstable case oscillates around a clearly nonzero mean value. The velocity field at $\mathrm{Re}=6000$ and $\mathrm{Re}=8500$ was averaged in time over one modal cycle (i.e., two piston cycles), yielding a flow $\langle\boldsymbol{V}\rangle_{t}$, which is dominantly azimuthal with only weak dependence on the axial coordinate $Z$. This may be related to the weakly nonlinear analysis of Racz, ${ }^{9}$ which indicates that the azimuthal flow at order $O(\epsilon)$ consists of quadratic mode-mode interaction terms and geostrophic components, the latter arising from forcing at $O\left(\epsilon^{2}\right)$. Based on their analysis, this azimuthal flow $u_{\theta}^{[2]}$ can be shown to be

$$
\begin{aligned}
V_{\theta}^{[2]}(r, Z, t)= & \epsilon|a|^{2}\left[U_{1}(r)+U_{2}(r) \sin \left(\omega_{0} t+\phi\right)\right. \\
& \left.+U_{3}(r) \cos \left(\frac{2 \pi Z}{h_{0}}\right)\right]
\end{aligned}
$$

where $a$ stands for the theoretical amplitude of the unstable mode $(1,1,0)$ and $U_{1}, U_{2}$, and $U_{3}$ are radial functions whose analytical expression is known. ${ }^{14}$ Whereas $U_{1}$ contains only geostrophic contributions, both $U_{2}$ and $U_{3}$ arise from modemode interactions. The presence of $U_{2}$ is removed by averaging in time. Figure 11 shows a comparison between $\epsilon|a|^{2} U_{1}(r)$ and the azimuthal flow $\left\langle V_{\theta}(r)\right\rangle_{t, Z}$ computed from our code, averaged in the $Z$ direction and defined by 

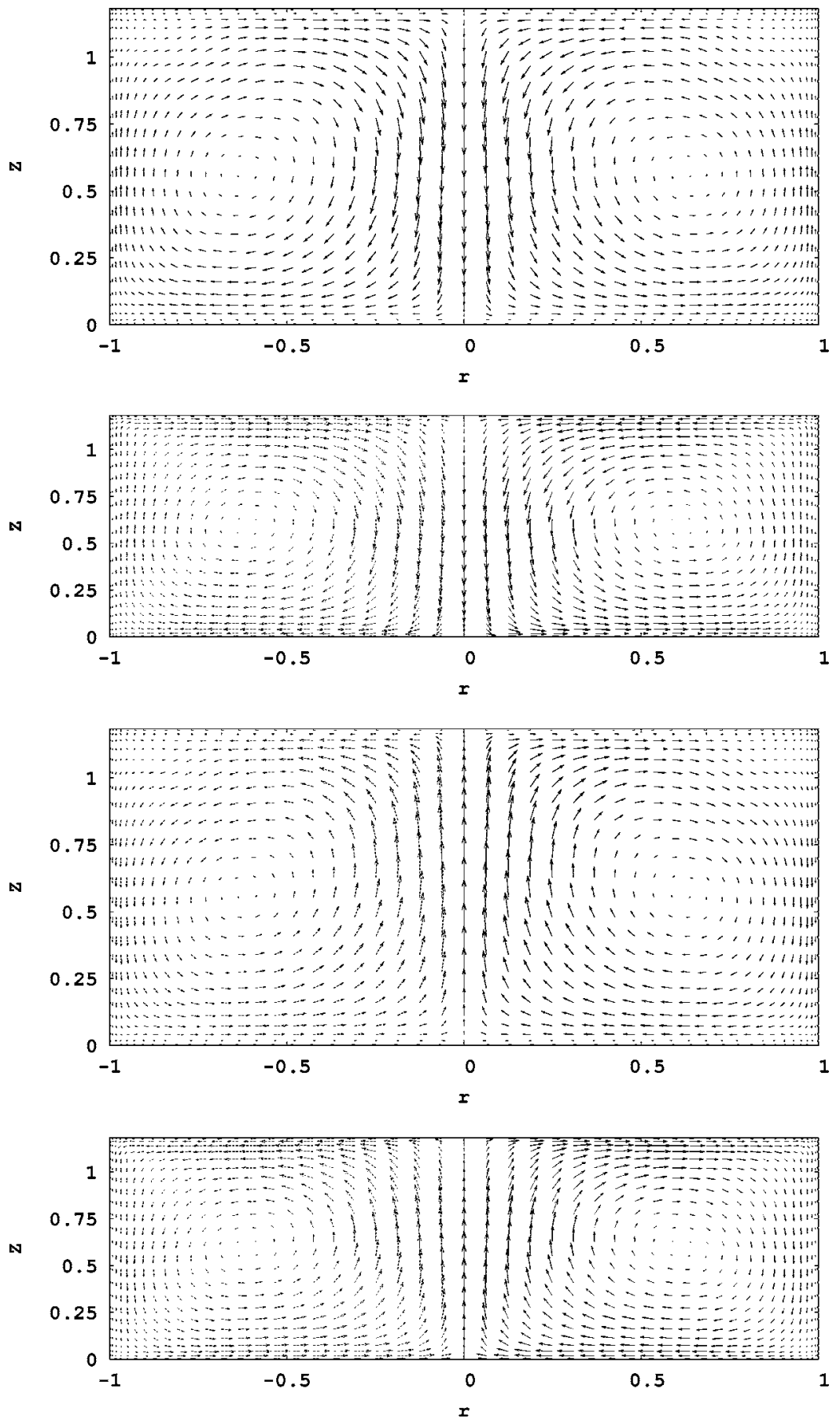

FIG. 8. Plane through the cylinder axis showing the velocity field filtered at angular frequency $\omega_{0} / 2, h_{0}$ $=1.18, \epsilon=0.131, \omega_{0}=1.15, \operatorname{Re}=8500$.

$$
\left\langle V_{\theta}\right\rangle_{t, Z}(r)=\frac{1}{h_{0}} \int_{0}^{h_{0}}\left\langle V_{\theta}\right\rangle_{t}(r, Z) d Z .
$$

Both azimuthal fields show a clear maximum near the cylinder axis, partially compensated by a deficit near the wall. The velocity excess predicted by the numerical code is more concentrated toward the axis, with a maximum located at $r=0.14$, whereas weakly nonlinear theory predicts this maximum at $r=0.3$. It is interesting to compare with the measured mean azimuthal velocity field obtained by Kobine. ${ }^{20}$ Although the inertial modes (whose nonlinear interaction generates the flow) are different, the results are similar: Kobine fits a power law of the form $r^{-c}$ in the range $0.1<r<0.5$ and interprets the result as showing a confined vortex flow. In our case, the nearly linear dependency of the azimuthal component as a function of $0.1<r<0.6$ in the simulation suggests a concentrated vortex near the axis and approximately uniform vorticity of opposite sign further out.

The axial dependence of $\langle\boldsymbol{V}\rangle_{t}$ is shown by plotting the difference $\left\langle V_{\theta}\right\rangle_{t}(r, Z)-\left\langle V_{\theta}\right\rangle_{t, Z}(r)$ and comparing with its theoretical counterpart $\left\langle u_{\theta}^{[2]}\right\rangle_{t}(r, Z)-\left\langle u_{\theta}^{[2]}\right\rangle_{Z, t}(r)$. As shown in Fig. 12 , there is a positive maximum near the midplane $Z=h_{0} / 2$ of the cylinder and negative values toward the top and bottom walls. In the theoretical case, this variation reflects the $\cos 2 \pi Z / h_{0}$ term in Eq. (32). A boundary layer is evident 

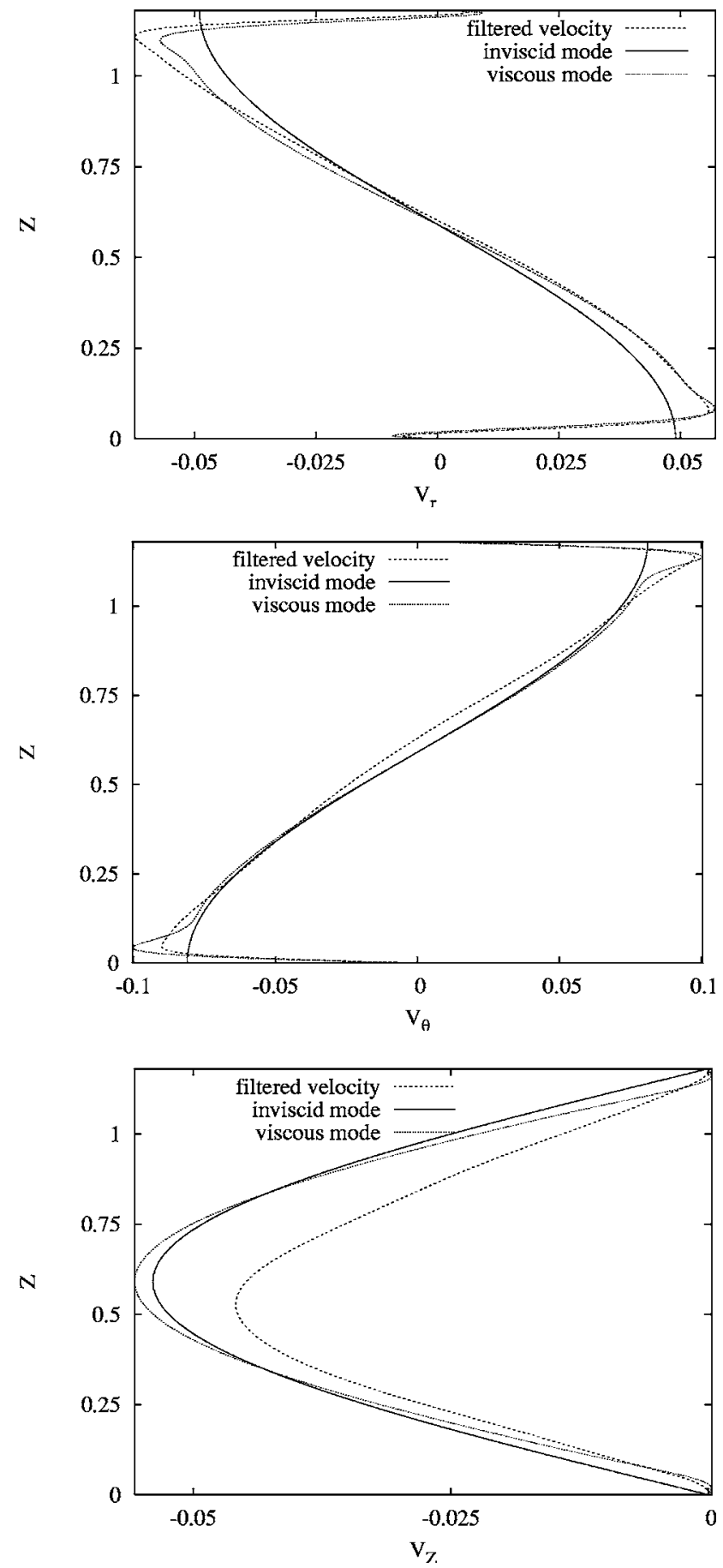

FIG. 9. Axial dependency of the velocity components of the saturated flow filtered at modal frequency, compared to the viscous and inviscid $(1,1,0)$ modes, at $r=0.4258 . h_{0}=1.18, \epsilon=0.131, \omega_{0}=1.15, \mathrm{Re}=6000$.

close to the top and bottom walls, characterized by an angular deviation of the velocity field, and preventing the timeaveraged flow from being purely azimuthal. Note that the departure between theory and simulation is larger than in the case of the leading-order flow. It may be useful to recall that the terms neglected in the asymptotic expansion (32) are only an order of magnitude $O\left(\epsilon^{1 / 2}\right)$ smaller than the retained terms. With $\epsilon^{1 / 2}$ close to 0.36 , this may explain the relatively large difference between theory and numerics.
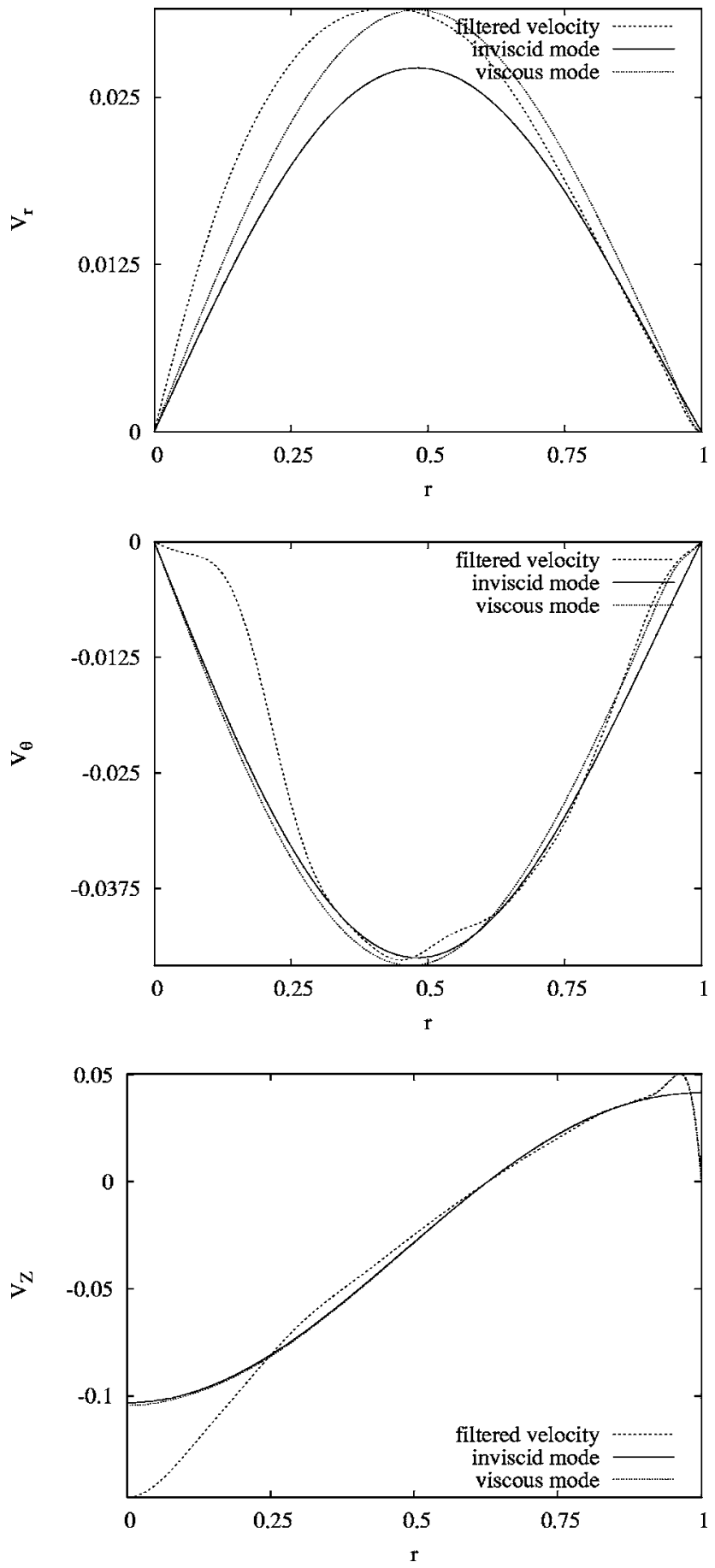

FIG. 10. Radial dependency of the velocity components of the saturated flow filtered at modal frequency, compared to the viscous and inviscid $(1,1,0)$ modes, at $Z=0.32 h_{0}, h_{0}=1.18, \epsilon=0.131, \omega_{0}=1.15, \mathrm{Re}=6000$.

\section{DISCUSSION}

We have performed an axisymmetric numerical simulation of the flow inside a finite rotating gas cylinder subject to periodic compression along its axis of revolution. The velocity singularities in the corner regions are treated by removing an analytically constructed singular flow that contains all the singularities of the flow, the remaining part of this decomposition being expanded on a Galerkin functional basis. In contrast to earlier analytical and experimental studies, numerical 
TABLE II. Modal energy factor of the filtered flow for $\mathrm{Re}=6000, \omega_{0}$ $=1.15$.

\begin{tabular}{lcccc}
\hline \hline$\%$ & $m=0$ & $m=1$ & $m=2$ & $m=3$ \\
\hline$\alpha=1$ & 0.008 & 93.6 & 0.019 & 0.51 \\
$\alpha=2$ & 0.009 & 1 & 0.39 & 0.1 \\
$\alpha=3$ & 0.013 & 0.48 & 0.16 & 0.08 \\
$\alpha=4$ & 0.0025 & 0.076 & 0.035 & 0.002 \\
\hline \hline
\end{tabular}

simulation offers an accurate way of exploring the near-wall viscous structure of the basic flow. This flow is found to be stable if the Reynolds number is low enough and/or if the system is far from resonance. Seen from the rotating frame, it consists of dominantly axial oscillations of the fluid. Viscous effects induce a boundary layer of thickness $O\left(\mathrm{Re}^{-1 / 2}\right)$. These results support the asymptotic analysis of Racz, ${ }^{9,14}$ who concluded that, for small $\epsilon$, viscous effects on the basic flow, essentially confined to the walls, have no influence on the instability of the overall flow at leading order.

Focusing on the instability of the axisymmetric inertial mode $(1,1,0)$, the simulations have confirmed the existence of a parametric instability, as shown by the marginal stability diagram in Fig. 6. This mode is found to be linearly unstable if the forcing frequency lies in a band centered around twice the modal frequency (which implies $\omega_{0}$ in the interval $[0: 4 \Omega]$ for instability, consistent with Mansour and Lundgren $^{8}$ ). Viscosity leads to an instability threshold whose value compares well with that given by theory. Numerical exploration of the instability has shown three different nonlinear regimes: (i) stability of the basic flow, (ii) instability of the basic flow leading to a saturated inertial mode with constant amplitude, and (iii) instability with weak periodic modulation of the unstable mode amplitude. The saturated case $\operatorname{Re}=6000, \omega_{0}=1.15$ was studied extensively, showing that the modal energy factor of the inviscid inertial mode $(1,1,0)$ is only a few percent less than unity.

This study has also shown, in the unstable case, the presence of a $O(\epsilon)$ azimuthal circulation resulting from nonlinear mode-mode interactions and geostrophic mode forcing. This circulation, observed to be positive near the cylinder axis and

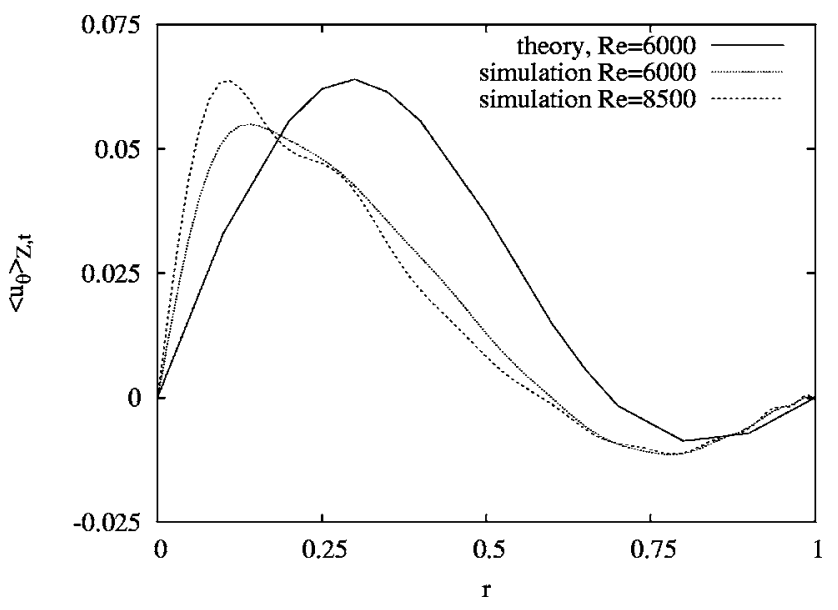

FIG. 11. Mean azimuthal velocity $\left\langle u_{\theta}\right\rangle_{t, Z}(r)$.

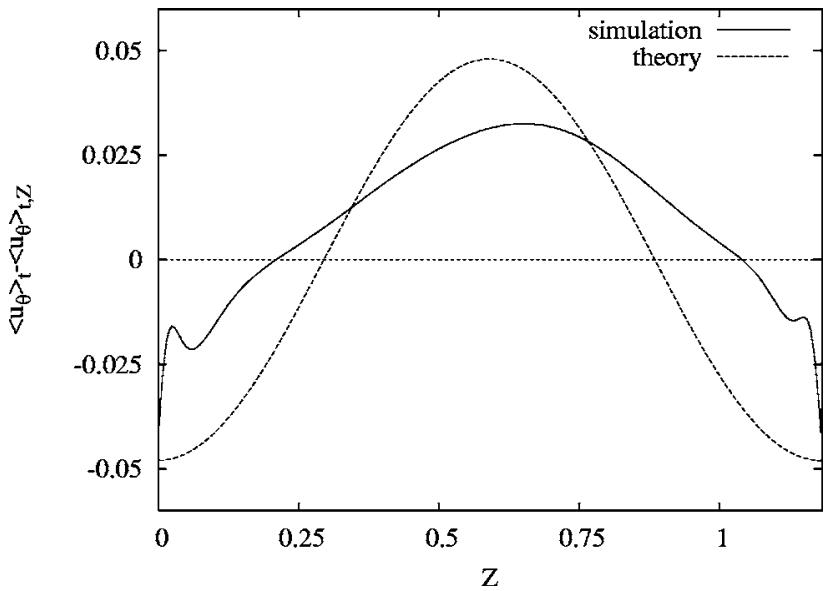

FIG. 12. Axial dependence of the departure $\left\langle u_{\theta}\right\rangle_{t}(r, Z)-\left\langle u_{\theta}^{[2]}\right\rangle_{Z, t}(r)$ at $r$ $=0.3$ for $\operatorname{Re}=6000$.

negative near the walls, is associated with axisymmetric geostrophic modes. The presence of geostrophic components giving rise to a nonzero mean azimuthal flow played an important role in Ref. 9 and has also been noted in the case of other instabilities based on resonant inertial-mode interactions, either of parametric type ${ }^{21}$ or due to resonant direct forcing. ${ }^{2,3,20,22}$ In these latter experimental studies, the azimuthal flow is observed to grow until breakdown occurs. This is not the case here where no breakdown is observed, and where the amplitude of the geostrophic flow fits well with the results of Racz. ${ }^{9}$ However, geostrophic modes do play a role in amplitude modulation that can be explained by the following qualitative scenario: ${ }^{2,9}$ beginning in the linear regime, the unstable mode pair grows and is affected by nonlinearity once its amplitude reaches $O\left(\epsilon^{1 / 2}\right)$, at which time the geostrophic flow has been driven to $O(\epsilon)$. The resulting $O(\epsilon)$ modification of the mean azimuthal velocity is sufficient to shift the natural frequency of the unstable mode by an amount comparable with the frequency bandwidth for instability. ${ }^{14}$ This resonant detuning can be enough to restabilize the flow.

The three different regimes observed numerically compare well with the experimental observations of Graftieaux ${ }^{11}$ in the case $\epsilon=0.131$. The dynamics of the flow seems rather less rich than predicted by the nonlinear equations of weakly nonlinear analysis. ${ }^{9}$ The most striking difference is that the amplitude modulations observed numerically are much weaker than the analytical predictions. As the distance from the instability threshold increases, Racz reports a growing modulation amplitude, and even possible exponential divergence (observed neither numerically nor experimentally). As the modulation increases, particularly just before divergence, it becomes so large that the asymptotic expansions are secular, with nominally higher-order terms comparable with leading order. When this happens, weakly nonlinear analysis is of course no longer applicable.

These results are reminiscent of other, previously studied, parametric instabilities giving rise to a subharmonic response to periodic forcing. Among them are the pendulum with vertically oscillating suspension and Faraday surface 
waves in a vertically oscillating tank. Closer to the context of periodically deformed rotating flows, the mechanism presented in this study is analogous to the elliptic instability with deformation applied along the rotation axis rather than in a plane orthogonal to it. The mode selection criterion is found to have much the same features and the action of viscosity is similar. ${ }^{6,7}$ However, in our case, the forcing frequency can be varied independently of the rotation rate, thus giving meaning to the notion of being more or less "far from resonance." Whereas the elliptic instability intrinsically involves nonaxisymmetric inertial modes, the configuration with axial periodic strain makes possible the excitation of purely axisymmetric modes. An interesting issue is whether an inertial mode, once excited, can be subject to a secondary instability involving other inertial contributions than the unstable mode itself and the geostrophic components. Mason and Kerswell ${ }^{23}$ showed numerically that a saturated nonaxisymmetric inertial mode is unstable to finite-amplitude perturbations, and related the resonant inertial triads to vortex breakdown commonly observed in elliptical configurations. In contrast to their study, the mode excited here is an axisymmetric one. Numerical simulation does not show secondary instability, but the axisymmetric nature of the algorithm prevents us from taking into account nonaxisymmetric perturbations. However, the inertial mode has been found experimentally to be stable for the same parameter values, from which we may conclude that it is not subject to secondary instability.

\section{ACKNOWLEDGMENT}

Y.D. would like to thank the French Ministère de l'Education Nationale, de la Recherche et de la Technologie for its support.

\section{APPENDIX: SOME ELEMENTS OF WEAKLY NONLINEAR THEORY}

In this appendix, we give a brief summary of the basic elements of the weakly nonlinear theory referred to in the paper. ${ }^{9}$ The velocity perturbation is expanded in terms of the inviscid Kelvin modes and exact equations derived for the modal amplitudes using modal orthogonality. These equations are intractable as they stand, but asymptotic analysis is possible in the limit of small $\epsilon$ and large Re (of order $\epsilon^{-2}$ ).

For simplicity sake, we restrict attention to the axisymmetric case studied in the paper. In that case, it is found that, to leading order, instability leads to growth of a single (primary) mode and its complex conjugate. The resonance condition, necessary but not sufficient for instability, is that the piston frequency, $\omega_{0}$, be order $\epsilon$ or less from twice the primary modal frequency, $2 \omega$. Modal time evolution is found to be affected significantly by nonlinearity once the primary modal amplitude grows to order $\epsilon^{1 / 2}$, hence asymptotic expansions for all modal amplitudes as power series in $\epsilon^{1 / 2}$ are used, with the leading-order term zero except for the primary mode and its conjugate.

At leading order and on a rapid-time scale, the primary mode simply oscillates with its inviscid, linear frequency, unaffected by piston motion. However, the (complex) oscillation amplitude is a function of the slow-time variable $T$ $=\epsilon t$ and an amplitude equation arises at order $\epsilon^{3 / 2}$. Prior to that, at order $\epsilon$, no secularity occurs, but other modes are present, including the geostrophic (zero-frequency) ones, which play an important role later. At order $\epsilon^{3 / 2}$, the slowtime primary amplitude equation appears as a nonsecularity condition and contains the infinity of $O(\epsilon)$ (real) geostrophic mode amplitudes, $A_{\sigma}$. Deriving equations for the latter requires going to still the next order, $\epsilon^{2}$.

The resulting system of equations has the form

$$
\frac{d a}{d T}=i \omega C a^{*}+\left(\frac{1}{2} i \Delta-D+2 i \sum_{\sigma} \Lambda_{\sigma} A_{\sigma}\right) a+i G|a|^{2} a,
$$

$$
\frac{d A_{\sigma}}{d T}=-D_{\sigma} A_{\sigma}+i C \hat{\Lambda}_{\sigma}\left(a^{2}-a^{* 2}\right)+\Gamma_{\sigma}|a|^{2},
$$

where $a$ and $a^{*}$ represent the primary mode amplitude and its complex conjugate, $\omega$ is the mode frequency, $C$ is a coupling coefficient due to piston motion, $\Delta$ is a parameter measuring

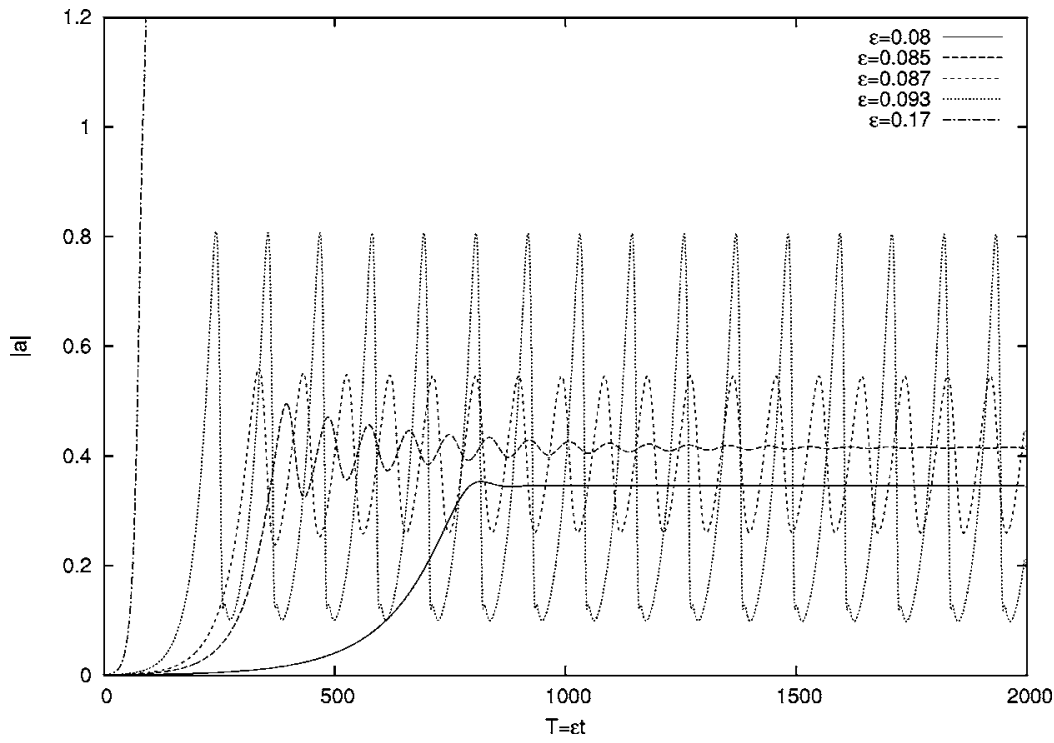

FIG. 13. Amplitude (modulus) of primary mode according to weakly nonlinear theory, $\operatorname{Re}=10^{4}, h_{0}=2$, $\omega_{0}=2 \omega^{(1,1,0)}$, for $\epsilon=0.08,0.085,0.087,0.093,0.17$. 


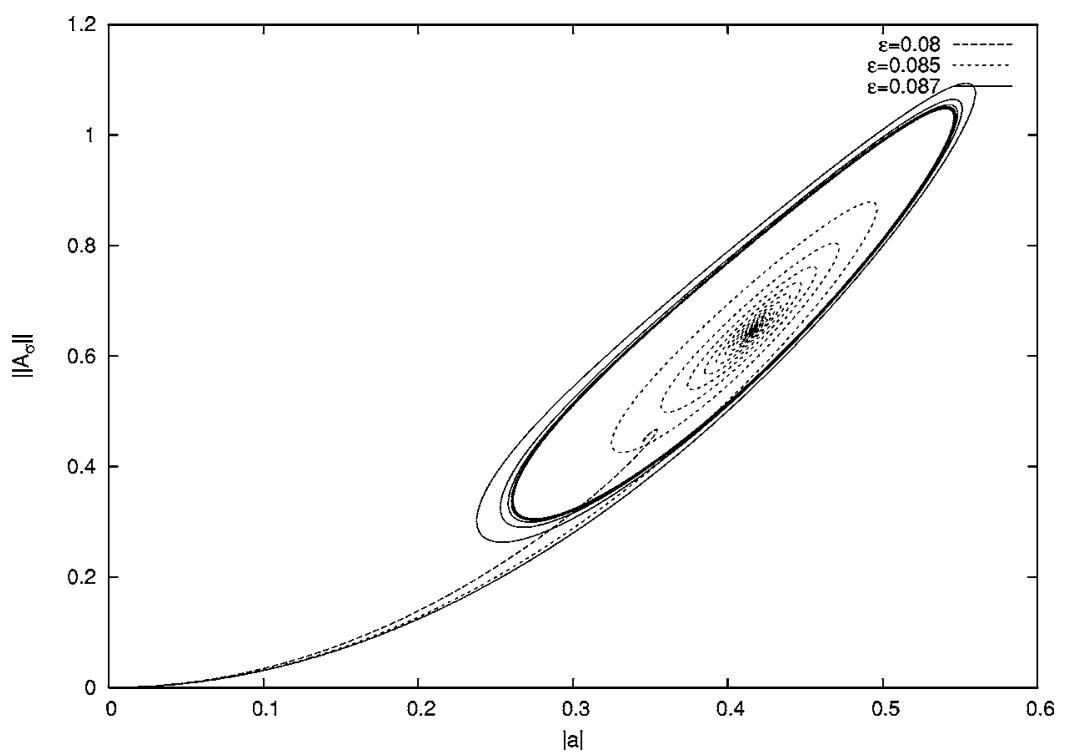

FIG. 14. Trajectory in the phase plane $\left(|a|,\left\|A_{\sigma}\right\|\right)$ according to weakly nonlinear theory, where $\left\|A_{\sigma}\right\|$ $=\left(\sum_{i=1}\left|A_{\sigma}\right|^{2}\right)^{1 / 2}, \quad \operatorname{Re}=10^{4}, h_{0}=2, \omega_{0}=2 \omega^{(1,1,0)}$, for $\epsilon$ $=0.08,0.085,0.087$.

the scaled departure of piston frequency from exact subharmonic resonance, and $D$ and $D_{\sigma}$ are viscous modal damping coefficients. All other terms are nonlinear: those in (A1) express detuning of the primary modal frequency by the geostrophic flow and cubic interactions of the primary mode with itself, while those in (A2) correspond to different types of quadratic forcing of the geostrophic modes by the primary mode. Comparatively simple analytical expressions for all coefficients in (A1) and (A2) have been derived, but are too lengthy to give here.

Dropping the nonlinear terms yields the linear theory in which the geostrophic modes do not appear in (A1), which is then easily analytically solved. A linear stability criterion results, leading to the neutral curve referred to in the text. In the nonlinear regime, numerical solution of the amplitude equations (truncating the infinity of geostrophic amplitudes—negligible differences being observed above about 10) shows different behavior depending on the parameter values: (a) saturation to constant $|a|$ and $A_{\sigma}$, with phase locking to the piston, (b) a periodic limit cycle, (c) apparently aperiodic large-time oscillations, or (d) exponential divergence of the modal amplitudes to infinity. Clearly, the latter behavior calls into question the weakly nonlinear approach used, as indeed do the large values of modal amplitudes found in some periodic and aperiodic cases.

Some sample results of numerical integration of the amplitude equations are shown in Figs. 13 and 14.

${ }^{1}$ Lord Kelvin, "Vibrations of a columnar vortex," Philos. Mag. 10, 155 (1880).

${ }^{2}$ A. D. Mac Ewan, "Inertial oscillations in a rotating cylinder," J. Fluid Mech. 40, 603 (1970).
${ }^{3}$ R. Manasseh, "Nonlinear behaviour of contained inertia waves," J. Fluid Mech. 315, 151 (1996).

${ }^{4}$ B. J. Bayly, "Three-dimensional instability of elliptical flow," Phys. Rev. Lett. 57, 2160 (1986).

${ }^{5} \mathrm{~F}$. Waleffe, "On the three-dimensional instability of strained vortices," Phys. Fluids A 2, 76 (1990).

${ }^{6}$ R. R. Kerswell, "Elliptical instability," Annu. Rev. Fluid Mech. 34, 83 (2002).

${ }^{7}$ C. Eloy, P. Le Gal, and S. Le Dizès, "Elliptic and triangular instabilities in rotating cylinders," J. Fluid Mech. 476, 357 (2003).

${ }^{8}$ N. N. Mansour and T. S. Lundgren, "Three-dimensional instability of rotating flows with oscillating axial strain," Phys. Fluids A 2, 2089 (1990).

${ }^{9}$ J. P. Racz, Ph.D. thesis, Ecole Centrale de Lyon, 2003.

${ }^{10}$ L. Graftieaux, L. Le Penven, J. F. Scott, and N. Grosjean, "A new parametric instability in rotating cylinder flow," in Advances in Turbulence IX, Proceedings of the 9th European Turbulence Conference, Southampton, UK, edited by I. P. Castro and P. E. Hancock (CIMNE, Barcelona, 2002).

${ }^{11}$ L. Graftieaux, Ph.D. thesis, Ecole Centrale de Lyon, 2003.

${ }^{12}$ H. P. Greenspan, The Theory of Rotating Fluids (Cambridge University Press, Cambridge, 1969).

${ }^{13}$ R. R. Kerswell and C. F. Barenghi, "On the viscous decay rates of inertial waves in a rotating cylinder," J. Fluid Mech. 285, 203 (1995).

${ }^{14}$ Y. Duguet, Ph.D. thesis, Ecole Centrale de Lyon, 2004.

${ }^{15}$ C. Canuto, Y. Hussaini, A. Quarteroni, and T. Zang, Spectral Methods in Fluid Dynamics (Springer, Berlin, 1988).

${ }^{16}$ G. K. Batchelor, An Introduction to Fluid Dynamics (Cambridge University Press, Cambridge, 1967).

${ }^{17}$ E. Serre and J. P. Pulicani, "A three-dimensional pseudospectral method for rotating flows in a cylinder," Comput. Fluids 30, 491 (2001).

${ }^{18}$ H. K. Moffatt, "Viscous and resistive eddies near a sharp corner," J. Fluid Mech. 18, 1 (1964).

${ }^{19} \mathrm{M}$. Fullekrug, Schumann-Resonanzen in den Magnetfeld-Komponenten (Cuvillier, Goettingen, 1994).

${ }^{20}$ J. J. Kobine, "Azimuthal flow associated with inertial wave resonance in a precessing cylinder," J. Fluid Mech. 319, 387 (1996).

${ }^{21}$ F. Waleffe, Ph.D. thesis, Massachusetts Institute of Technology, 1989.

${ }^{22}$ J. J. Kobine, "Inertial wave dynamics in a rotating and precessing cylinder," J. Fluid Mech. 303, 233 (1995).

${ }^{23}$ D. M. Mason and R. R. Kerswell, "Nonlinear evolution of the elliptical instability: An example of inertial wave breakdown," J. Fluid Mech. 396, 73 (1999). 